for the effects of passive smoking on smokers. Therefore the main emphasis of this paper is an estimation of the risks of passive smoking in lifelong non-smokers; data are presented for the active smoking groups to provide an estimate of dose-response.

Our results are based on a general population cohort study carried out in an area with a high level of diseases related to smoking. A consistent increase in risk was observed in passive smokers for each of the 10 variables measured covering respiratory symptoms, forced expiratory volume in one second, cardiovascular symptoms, and subsequent mortality, including lung cancer and ischaemic heart disease. A dose-response relation was seen, and the risks were biologically plausible in relation to the size of the risks found for the active smokers. These three factors taken together increase our concern that exposure to other people's tobacco smoke cannot be regarded as a safe involuntary practice.

1 Colley JRT, Holland WW, Corkhill RT. Influence of passive smoking and parental phlegm on pneumonia and bronchitis in early childhood. Lancet 1974;ii: 1031-4.

2 Weiss ST, Tager IB, Speizer FE, Rosner B. Persistent wheeze-its relation to respiratory illness, cigarette smoking, and level of pulmonary function in a respiratory illness, cigarette smoking, and level of pulmonary func

3 White JR, Froeb HF. Small airways dysfunction in non-smokers chronically exposed to tobacco smoke. $N$ Engl F Med 1980;302:720-3.

4 Kauffmann F, Tessier JF, Oriol W. Adult passive smokers in the home environment: a risk factor for chronic airflow limitation. Am F Epidemiol 1983;117:269-80

Lebowitz MD. Influence of passive smoking on pulmonary function: a survey Prev Med 1984;13:645-55.

6 Wald NJ, Nanchalal K, Thompson SG, Cuckle HS. Does breathing other people's tobacco smoke cause lung cancer? Br Med $\mathcal{f}$ 1986;293:1217-22.

7 Garland C, Barrett-Conner E, Suarez L, et al. Effects of passive smoking on ischaemic heart disease mortality of non-smokers: a prospective study. Am f Epidemiol 1985;121:645-50.
8 Hirayama T. Passive smoking: a new target of epidemiology. Tokai $f$ Exp Clin Med 1985;10:287-93.

9 Svendsen KH, Kuller LH, Martin MJ, Ockene JK. Effects of passive smoking in the multiple risk factor intervention trial. Am $\mathcal{F}$ Epidemiol 1987;126: $783-95$

10 US Department of Health and Human Services. Respiratory effects of involuntary smoke exposure: epidemiologic studies. Report of a workshop, 1-3 Mav 1983. Bethesda, Maryland: National Institutes of Health, 1983.

11 Gillis CR, Hole DJ, Hawthorne VM, Boyle P. The effect of environmental tobacco smoke in two urban communities in the west of Scotland. Eur $\mathcal{F}$ tobacco smoke in two urban commu
Respir Dis 1984;65 (suppl 133):121-6.

12 Hawthorne VM, Gillis CR, Maclean DS. Monitoring health in Scotland. Int $f$ Epidemiol 1975;1:369-74.

13 Hawthorne VM, Greaves DA, Beevers DG. Blood pressure in a Scottish town. BrMed F 1974;1:600-3.

14 Kaplan EL, Meier P. Nonparametric estimation from incomplete observations. fournal of the American Statistical Association 1958;53:457-81.

15 Cox DR. The analysis of binary data. London: Methuen, 1970.

16 Cox DR. Regression models and life tables. Fournal of the Royal Statistical Society $(B)$ 1972;34:187-220.

17 Cox DR. Partial likelihood. Biometrika 1975;62:269-76.

8 Dixon WJ, Brown MB, Engelman L, et al. Biomedical data processing programs. Statistical software 1985. Los Angeles: University of Calfornia Press, 1985.

19 Matsukura S, Taminato $T$ Kitano $N$, et al. Effects of environmental tobacco smoke on urinary cotinine excretion in non-smokers. N Engl $\mathcal{F} \mathrm{Med}$ 1984;311:828-32.

20 Hoffmann D, Brunnemann KD, Adams JD, et al. Indoor pollution by tobacco smoke: model studies on the uptake by non-smokers. In: Berglund B, ed. Indoor air, radon, passive smoking, particulates and housing epidemiology. Indoor air, radon, passive smoking, particulates and housing epidemiology.
Vol 2. Stockholm: Swedish Council for Building Research, 1984:313-8. Vol 2. Stockholm: Swedish Council for Building Research, $1984: 313-8$.
(Proceedings of the 3rd international conference on indoor air quality and climate, suppl D17.

21 Wald N, Ritchie C. Validation of studies on lung cancer in non-smokers married to smokers. Lancet 1984;i: 1067 .

22 Gillis CR, Hole DJ, Hawthorne VM. Cigarette smoking and male lung cancer in an area of very high incidence. II. Report of a general population cohor study in the West of Scotland. F Epidemiol Community Health 1988;42:44-8.

23 Lee PN. Misclassification as a factor in passive smoking risk. Lance 1986;ii:867.

24 Burch PRJ. Passive smoking in adulthood and cancer risk. Am $\mathcal{f}$ Epidemiol 1986;123:368-9.

25 Wald NJ, Boreham J, Bailey A, Ritchie C, Haddow JE, Knight G. Urinary cotinine as a marker of breathing other people's tobacco smoke. Lance 1984;i:230-1.

\title{
Carbohydrate deficient transferrin: a marker for alcohol abuse
}

\author{
A Kapur, G Wild, A Milford-Ward, D R Triger
}

\section{Abstract}

Objective-To assess the value of serum carbohydrate deficient transferrin as detected by isoelectric focusing on agarose as an indicator of alcohol abuse.

Design-Coded analysis of serum samples taken from patients with carefully defined alcohol intake both with and without liver disease. Comparison of carbohydrate deficient transferrin with standard laboratory tests for alcohol abuse.

Setting - A teaching hospital unit with an interest in general medicine and liver disease.

Patients-22 "Self confessed" alcoholics admitting to a daily alcohol intake of at least $80 \mathrm{~g}$ for a minimum of three weeks; 15 of the 22 self confessed alcoholics admitted to hospital for alcohol withdrawal; 68 patients with alcoholic liver disease confirmed by biopsy attending outpatient clinics and claiming to be drinking less than $50 \mathrm{~g}$ alcohol daily; 47 patients with non-alcoholic liver disorders confirmed by biopsy; and 38 patients with disorders other than of the liver and no evidence of excessive alcohol consumption.

Intervention-Serial studies performed on the 15 patients undergoing alcohol withdrawal in hospital.

Main outcome measure-Determination of relative value of techniques for detecting alcohol abuse.

Results-Carbohydrate deficient transferrin was detected in 19 of the $22(86 \%)$ self confessed alcohol abusers, none of the 47 patients with non-alcoholic liver disease, and one of the $38(3 \%)$ controls. Withdrawal of alcohol led to the disappearance of carbohydrate deficient transferrin at a variable rate, though in some subjects it remained detectable for up to 15 days. Carbohydrate deficient transferrin was considerably superior to the currently available conventional markers for alcohol abuse.

Conclusion-As the technique is fairly simple, sensitive, and inexpensive we suggest that it may be valuable in detecting alcohol abuse.

\section{Introduction}

The medical and social consequences of alcohol abuse are major problems throughout the world. Although many people readily acknowledge the extent of their alcohol consumption, others attempt to conceal it, and we lack reliable objective means of identifying surreptitious alcohol consumption. Currently available laboratory markers have considerable limitations, being insensitive, non-specific, or dependent on liver damage. The mean corpuscular volume rises in patients with thyroid disease, folic acid deficiency, and liver disease, ${ }^{1}$ whereas serum $\gamma$-glutamyltransferase activity is affected by drugs that induce microsomal enzymes as well as rising in all forms of obstructive liver damage. ${ }^{2}$ Serum aspartate aminotransferase activity is more commonly raised in alcoholics than alanine aminotransferase activity is, and whereas a ratio of aspartate to alanine aminotransferase activity of greater than 2:1 is strongly suggestive of alcoholic liver diseas $^{3}$ this is of little value in subjects in whom the 
aspartate aminotransferase activity remains within the normal range. Measurement of blood or urine ethanol concentration is clearly a specific test but is useful only if done within 12-24 hours after the last alcoholic drink and gives no assessment of the overall extent of alcohol abuse. ${ }^{2}$

In 1976 Stibler and Kjellin reported qualitatively abnormal transferrin in the serum of patients with alcoholic cerebellar tremor. ${ }^{4}$ Subsequent reports showed this transferrin to be depleted in sialic acid residues and confirmed its presence to be both sensitive and specific to alcohol abuse. ${ }^{56}$ We report a fairly simple technique for detecting this carbohydrate deficient transferrin and present our experience of its application in clinical practice. We determined the specificity and sensitivity of the abnormal transferrin for alcohol abuse and compared the method with others using some of the currently available biochemical markers.

\section{Patients and methods} PATIENTS

Five groups of subjects were chosen for study.

"Self confessed" alcoholics $(n=22)$ were all patients who admitted readily to regular alcohol consumption of at least $80 \mathrm{~g}$ a day for a minimum of three weeks before the study. In almost every case the history was independently corroborated from other sources such as friends or relatives. All patients had undergone a percutaneous liver biopsy as part of their medical assessment (unrelated to this study) to determine the extent and nature of liver damage. Nine patients had fatty change, eight cirrhosis, and five alcoholic hepatitis without cirrhosis.

Alcoholics admitted to hospital for alcohol withdrawal $(n=15)$-These patients were also in the group of self confessed alcoholics. All were admitted to hospital for treatment either of their alcohol dependency or for decompensated liver disease secondary to alcohol abuse. All had either positive blood alcohol determinations or objective evidence of alcohol ingestion (alcohol on the breath or clinically intoxicated) at the time of admission to hospital. Patients were admitted to a short stay medical gastroenterology ward, where every precaution was taken to prevent the reintroduction of alcohol. Serial blood samples were taken two or three times a week to detect transferrin.

Outpatients with alcoholic liver disease $(n=68)$ that had been confirmed by biopsy who were attending an outpatient liver clinic were questioned carefully by an

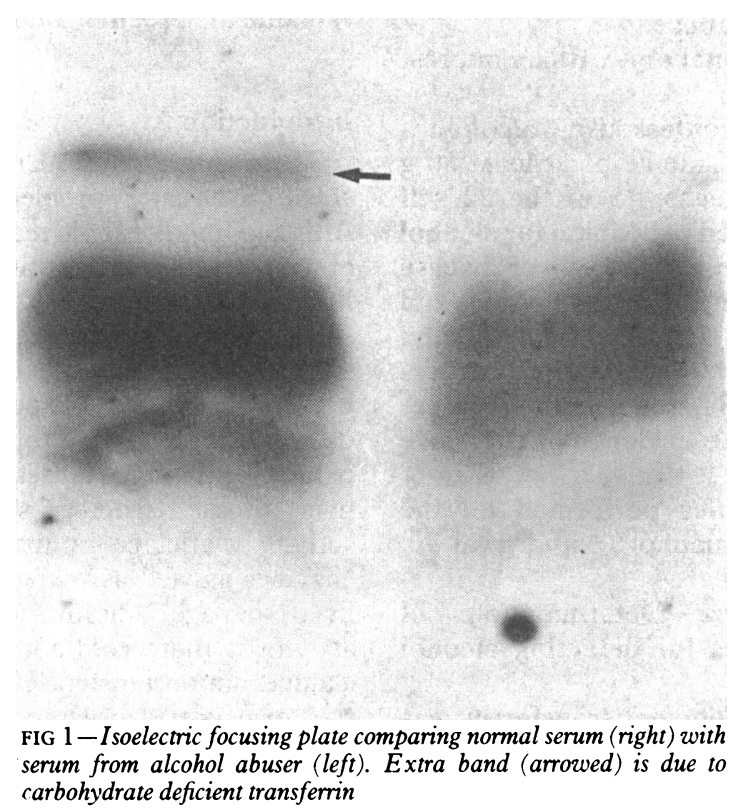

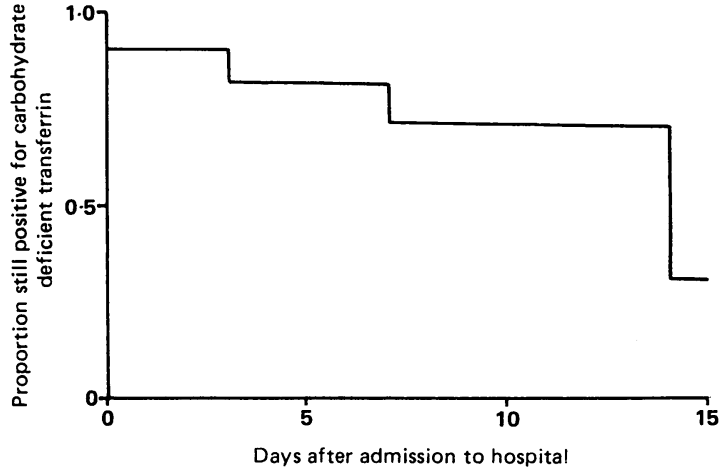

FIG 2-Estimated proportion of 15 patients undergoing alcoho withdrawal still positive for carbohydrate deficient transferrin at various times after admission to hospital (Kaplan-Meier life table analysis)

experienced clinician about their current and recent alcohol intake. Only patients who admitted to an alcohol intake of $<50 \mathrm{~g}$ daily were included in this group. Forty eight patients had established cirrhosis, nine steatosis, and six alcoholic hepatitis. No subject in this category belonged to the first two groups.

Patients with non-alcoholic liver disease $(n=47)-$ Serum samples were taken from patients with a variety of non-alcoholic liver disorders. All of these disorders were confirmed histologically except in three patients with liver damage related to paracetamol, in whom the diagnosis was made on clinical and biochemical grounds alone. All patients were asked specifically about alcohol consumption and those with a daily intake of $>20 \mathrm{~g}$ were excluded.

Controls without liver disease $(n=38)$ were an unselected group of patients attending a general medical outpatient clinic. None had evidence of primary liver disease, though many had abnormal results of liver biochemical tests due to their illness. Patients were specifically questioned about alcohol consumption and only those admitting to $<20 \mathrm{~g}$ a day were included.

\section{LABORATORY INVESTIGATIONS}

All patients had blood samples taken to detect carbohydrate deficient transferrin, for blood count, and for liver biochemistry profile. To test for carbohydrate deficient transferrin blood was taken into a sterile tube and allowed to clot before being centrifuged at $3000 \mathrm{rpm}$. The serum was stored at $-20^{\circ} \mathrm{C}$ before testing. Samples were tested under code, and all tests were carried out within three months after collection of the serum.

Routine laboratory markers for alcohol abuse that were investigated were mean corpuscular volume, ratio of aspartate to alanine aminotransferase activity, and $\gamma$-glutamyltransferase activity. Mean corpuscular volume was estimated with a continuous flow automated haematology analyser (Technicon H 6000, Technicon Instruments, Basingstoke) with a sample of citrated blood. The remaining tests were performed with a multichannel autoanalyser (Technicon SMAC, Technicon Instruments, Basingstoke). Blood alcohol determinations were carried out with headspace gas liquid chromatography analysis. ${ }^{7}$

Abnormal results were defined as those that fell outside the normal reference range for the laboratory. These were $>98 \mathrm{fl}$ for mean corpuscular volume ${ }^{8}$ and $>45 \mathrm{U} / \mathrm{l}$ for $\gamma$-glutamyltransferase activity.

\section{CARBOHYDRATE DEFICIENT TRANSFERRIN}

Carbohydrate deficient transferrin was identified by isoelectric focusing in agarose $\mathrm{pH} 3-10$, with the transferrin bands being positively identified by immunofixation. Sorbitol $(3.6 \mathrm{~g})$ and agarose $(0.3 \mathrm{~g})$ (Pharmacia UK, Milton Keynes) were dissolved in 
$27 \mathrm{ml}$ distilled water by boiling. The resultant solution was cooled to $75^{\circ} \mathrm{C}$ and $2 \mathrm{ml}$ ampholine solution pH 3-10 (Pharmalyte, Pharmacia UK) added and mixed thoroughly. Gels were cast on to Gelbond film (Pharmacia UK) in a frame. When set the gels were removed from the casting frame and allowed to equilibrate in a humidity chamber at $4^{\circ} \mathrm{C}$ for 15 minutes.

Serum samples diluted one part in 10 in distilled water were applied in $5 \mathrm{ml}$ loads to the cathodic side of the gel with an application foil. The cathode was formed with $1 \mathrm{M}$ sodium hydroxide and the anode with $0.05 \mathrm{M}$ sulphuric acid. Electrofocusing was carried out at $10^{\circ} \mathrm{C}, 1000 \mathrm{~V}, 120 \mathrm{~mA}$, and $15 \mathrm{~W}$ for three hours, the sample application foil being removed after 45 minutes. After focusing the gel was immunofixed with nonspecific antihuman transferrin (Protein Reference Unit, Central Antiserum Procurement Unit, Sheffield) and incubated at $37^{\circ} \mathrm{C}$ for one to two hours in a humidity chamber. The gel was then washed in saline overnight and in distilled water for two hours before drying. Transferrin bands were visualised by staining with Coomassie brilliant blue G (PAGE blue, BDH, Poole).

Positive samples were defined as those in which an additional transferrin band was delineated at $\mathrm{pI} 5 \cdot 7$ (fig 1). Occasionally, gels failed to give a clear pattern owing to poor resolution. Such samples were retested and classified as uninterpretable only if the second test produced similar results.

\section{STATISTICAL ANALYSIS}

The ability of carbohydrate deficient transferrin and the other biochemical markers to discriminate between alcoholic and non-alcoholic liver disease was assessed by $\chi^{2}$ test, with Yates's correction when appropriate. The persistence of carbohydrate deficient transferrin

TABLE I-Carbohydrate deficient transferrin in patients with alcoholic and non-alcoholic liver disease

\begin{tabular}{|c|c|c|c|c|}
\hline & $\begin{array}{l}\text { Self confessed } \\
\text { alcoholics }\end{array}$ & $\begin{array}{l}\text { "Abstinent" } \\
\text { alcoholics }\end{array}$ & $\begin{array}{l}\text { Patients with } \\
\text { non-alcoholic } \\
\text { liver disease }\end{array}$ & Controls \\
\hline $\begin{array}{l}\text { Carbohydrate deficie } \\
\text { Present } \\
\text { Absent } \\
\text { Uninterpretable }\end{array}$ & $\begin{array}{l}19(86 \%) \\
2 \\
1\end{array}$ & $\begin{array}{l}8(12 \%) \\
55 \\
5\end{array}$ & $\begin{array}{r}46 \\
1\end{array}$ & $\begin{array}{l}1(3 \%) \\
36^{1}\end{array}$ \\
\hline Total & 22 & 68 & 47 & 38 \\
\hline
\end{tabular}

«Uninterpretable results are equated with negative results.

Ability of carbohydrate deficient transferrin to discriminate between self confessed (actively drinking) alcoholics and non-alcoholic subjects: $\chi^{2}=63 \cdot 8, \mathrm{df}=1, \mathrm{p}<0 \cdot 0001$.

TABLE II-Abnormal determination of mean corpuscular volume and $\gamma$-glutamyltransferase and ratio of aspartate to alanine aminotransferase activity in alcoholics and non-alcoholic patients

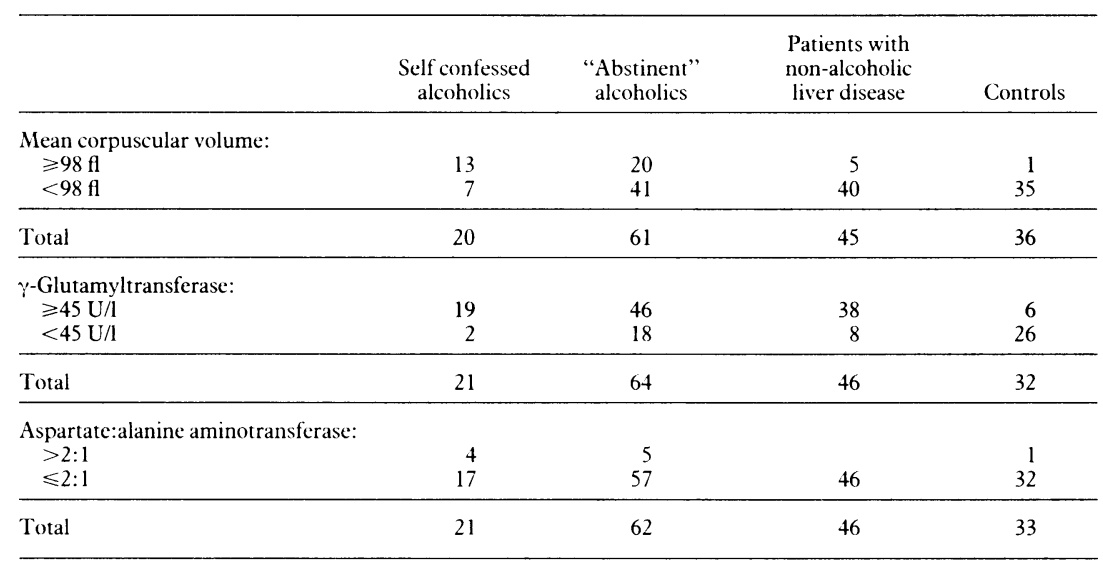

Ability of mean corpuscular volume to discriminate between self confessed (actively drinking) alcoholics and non-alcoholic subjects $(\mathbf{n}=71): \chi^{2}=27 \cdot 9, \mathrm{df}=1, \mathrm{p}<0 \cdot 001$

Ability of mean $\gamma$-glutamyltransferase activity to discriminate between self confessed (actively drinking) alcoholics and all non-alcoholic patients $(\mathrm{n}=78): \gamma^{2}=6 \cdot 47, \mathrm{df}=1, \mathrm{p}=0 \cdot 02$; all non-alcoholics with liver disease $(\mathrm{n}=46)$ : $\gamma^{2}=0 \cdot 225, \mathrm{df}=1, \mathrm{p}>0 \cdot 6 ;$ controls $(\mathrm{n}=32): \chi^{2}=21 \cdot 9, \mathrm{df}=1, \mathrm{p}<0 \cdot 0001$.

Ability of aspartate:alanine aminotransferase activity to discriminate between self confessed (actively drinking) alcoholics and non-alcoholic subjects $(n=79): \chi^{2}=11 \cdot 37, \mathrm{df}=1, \mathrm{p}<0 \cdot 001$. after alcohol withdrawal was expressed with KaplanMeier life table analysis. ${ }^{9}$

\section{Results}

Table I shows the incidence of carbohydrate deficient transferrin in the different groups. Carbohydrate deficient transferrin was present in the serum of 19 of the $22(86 \%)$ self confessed alcoholics. In one patient the isoelectric pattern was uninterpretable whereas in two others there was no evidence of an abnormal band. These two patients both gave a history of daily alcohol intake of no more than $80 \mathrm{~g}$. In contrast, no positive results were found in the 47 patients with non-alcoholic liver disease and only one positive result in the 38 controls with a variety of non-hepatic disorders. In each of these groups, however, there was a single sample in which the transferrin bands could not be interpreted. Eight positive results were obtained from the 68 outpatient alcoholics admitting to an intake of $<50 \mathrm{~g}$ of alcohol per day: 55 samples were negative, and five could not be interpreted. Clinical and biochemical variables in the eight patients positive for carbohydrate deficient transferrin did not differ considerably from those in the 55 patients negative for it. In seven patients tested for $\gamma$-glutamyltransferase activity who were positive for transferrin all had high activities compared with 40 out of 52 patients negative for transferrin; the mean $\gamma$-glutamyltransferase activity was almost the same $(172 v 177 \mathrm{U} / \mathrm{l})$.

Serial studies on the 15 alcoholics withdrawing from alcohol showed persistence of the abnormal transferrin to be variable, ranging from one to 17 days after admission to hospital (fig 2). These data should be interpreted with caution as in most cases carbohydrate deficient transferrin was still present at the time of discharge from hospital or death. In contrast, blood alcohol was undetectable in five patients at the time of admission to hospital and in nine it became undetectable within 48 hours after admission. The remaining patient had a serum alcohol concentration of $75 \mathrm{mmol} / \mathrm{l}$ on admission; serial estimations showed such an exceptionally slow rate of clearance that ethanol had not disappeared from the blood until 72 hours after admission, and carbohydrate deficient transferrin was detectable until 15 days after admission.

Carbohydrate deficient transferrin was detectable in the serum of patients with established cirrhosis, alcoholic hepatitis, and fatty liver alone.

Table II shows the incidence of abnormal $\gamma$ glutamyltransferase activity, mean corpuscular volume, and ratio of aspartate to alanine aminotransferase activity in the self confessed alcoholics, outpatient alcoholics, non-alcoholics, and controls. Not all laboratory results were available for every patient in whom carbohydrate deficient transferrin was determined because of technical errors. Whereas $\gamma$-glutamyltransferase activity discriminated between alcohol abusers and controls, it did not distinguish alcohol abusers from patients with non-alcoholic liver disease. The mean corpuscular volume was only occasionally abnormal in the controls and patients with non-alcoholic liver disease, but it was high in only 13 out of $20(65 \%)$ of the alcohol abusers. The ratio of aspartate to alanine aminotransferase activity was rarely greater than 2:1 in non-alcoholic subjects, but it detected only four out of $21(19 \%)$ of the alcoholics. Simultaneous blood alcohol estimates were available in 24 of the 27 alcoholics who were positive for carbohydrate deficient transferrin. It was undetectable in six of these as well as in one of two self confessed alcoholics negative for carbohydrate deficient transferrin (no sample was available on the other).

Table III shows the sensitivity and observed 
specificity of the abnormal carbohydrate deficient transferrin compared with the other variables. The percentage of both true positive and true negative results with carbohydrate deficient, transferrin was higher than for any other test. The outpatient alcoholics were excluded from these calculations.

TABLE III-Sensitivity and specificity of carbohydrate deficien transferrin and other markers of alcohol abus

\begin{tabular}{lcc}
\hline & & Observed \\
& Sensitivity & specificity \\
\hline Carbohydrate deficient transferrin & $90 \cdot 5$ & $98 \cdot 8$ \\
Mean corpuscular volume & $65 \cdot 0$ & $92 \cdot 6$ \\
$\gamma$-Glutamyltransferase activity & $90 \cdot 5$ & $41 \cdot 0$ \\
Ratio of aspartate to alanine aminotransferase $>2: 1$ & $19 \cdot 0$ & $98 \cdot 7$ \\
\hline
\end{tabular}

\section{Discussion}

We have confirmed the results of earlier studies that carbohydrate deficient transferrin is valuable in identifying patients drinking excessive amounts of alcohol and have also shown its potential value in clinical medical practice. These findings are at variance with the study of Poupon et al, who found no significant difference in the pI 5.7 fraction of transferrin between alcoholic and non-alcoholic patients with cirrhosis. ${ }^{10}$ Their study, however, does not seem to have considered that abnormal transferrin disappears after alcohol withdrawal as there are no data on the time of testing in relation to alcohol consumption. False positive results are rarely found whereas most subjects consuming an average of $80 \mathrm{~g}$ of alcohol or more a day for at least three weeks showed a positive result irrespective of the nature or severity of the liver damage. Furthermore, the fact that the abnormal carbohydrate deficient transferrin remained detectable in the serum for several days after withdrawal of alcohol suggests that the test is superior to random blood alcohol determination (the only test hitherto available showing comparable specificity) in detecting abuse. Stibler et al reported four false positive results among 177 controls and noted that all were in elderly women." Interestingly, our single positive result among controls was in a 69 year old woman with chronic obstructive airways disease. No satisfactory information has been advanced for this apparent abnormality in elderly women.

The mechanism whereby the abnormal transferrin is produced is unknown. The electrophoretic mobility of transferrin seems to be altered by conditions in which the sialic acid residues on the carbohydrate terminals of the glycoprotein side chains of the transferrin molecule are altered. As the amounts of both sialic acid and carbohydrate depletion are uncertain we prefer the term "carbohydrate deficient" to desialylated transferrin.

In keeping with earlier observations our experience suggests that a daily consumption of $80 \mathrm{~g}$ of alcohol is required to produce a positive result. Although this is somewhat higher than the $60 \mathrm{~g}$ and $40 \mathrm{~g}$ recommended as being safe amounts for men and women respectively, ${ }^{12}$ it nevertheless provides a means for detecting a substantial proportion of people who are at major risk from the harmful effects of alcohol. An estimated 400000 people in the United Kingdom drink 80 g or more alcohol daily. ${ }^{13}$

The value of carbohydrate deficient transferrin as a screening test is arguably restricted by the fact that it cannot be quantified. Our serial studies have shown carbohydrate deficient transferrin to be either present or absent by isoelectric focusing. Our attempts to quantify the test by varying the conditions of electrophoresis failed to produce satisfactory results. Storey et al attempted to quantify the test by expressing the desialylated transferrin in relation to total transferrin." Their data do not suggest that this is superior to the carbohydrate deficient transferrin that we measured and, though results may be obtained within a few hours, only one sample can be analysed at a time. Although serum samples may be safely transported, they should not be left at room temperature for more than two days before testing as this leads to shifts in the transferrin spectrum, probably due to the effect of naturally occurring bacterial neuraminidase. ${ }^{14}$ We have also found that false positive results may be obtained if samples are stored at $-20^{\circ} \mathrm{C}$ for 12 months or longer.

We found carbohydrate deficient transferrin to be more sensitive and specific than the more commonly used laboratory markers of alcohol abuse and probably superior to other tests that have been advocated. Chalmers et al advocated a combination of mean corpuscular volume, $\gamma$-glutamyltransferase activity, and alkaline phosphatase activity, which yields a $70 \%$ specificity and $85 \%$ sensitivity. ${ }^{15}$ Not only is this less specific than the abnormal carbohydrate deficient transferrin but it also requires several different laboratory determinations, thereby increasing the cost. We attempted to apply the formula to our patients and found it unhelpful, probably because of differences in the normal values for mean corpuscular volume and $\gamma$-glutamyltransferase activity, which do not have international standards. The need for each laboratory to devise its own formula on the basis of local standard values would make this mathematical model even less attractive for clinical use. More recently, mitochondrial aspartate aminotransferase activity and the ratio of mitochondrial to total aspartate aminotransferase activity have been advocated as tests for alcoholism, but both fail to distinguish between alcoholic and non-alcoholic liver damage. ${ }^{16}$

Our preliminary (unpublished) experience led us to use isoelectric focusing in agarose in preference to polyacrylamide as described by Stibler et al. ${ }^{17}$ This technique enables a permanent record to be obtained. Results can be obtained within 72 hours after receipt of the sample, and up to 20 samples at a time may be tested on each gel. Fairly inexpensive equipment and reagents are required and, even if allowance is made for the time of the technician, the cost of the test compares favourably with that of other standard laboratory investigations. Although occasional results are obtained that are not interpretable even on retesting, the sensitivity and specificity of carbohydrate deficient transferrin seem to be so superior to those of currently available tests that we consider it to be an important advance in the detection of alcohol abuse.

We thank Dr R A Dixon, department of community medicine, University of Sheffield, for statistical advice.

1 Whitfield JB, Hensley WJ, Bryden D, Gallagher H. Some laboratory correlates of drinking habits. Am Clin Biochem 1978;15:297-303.

2 Lewis KO, Paton A. Tools of detection. Br Med f 1981;283:1531-2.

3 Skrede S, Blomhoff JP, Gjone E. Biochemical features of acute and chronic hepatitis. Ann Clin Res 1976;8:182-99.

4 Stibler H, Kjellin KG. Isoelectric focusing and electrophoresis of the CSF protein in tremor of different origins. F Neurol Sci 1976;30:269.

5 Stibler $\mathrm{H}$, Borg S, Allgulander C. Clinical significance of abnormal heterogeneity of transferrin in relation to alcohol consumption. Acta Med Scand 1979;206:275-81.

6 Storey EL, Anderson GJ, Mack U, Powell LW, Halliday JW. Desialylated transferrin as a serological marker of chronic excessive alcohol ingestion. Lancet 1987; i: 1292-6.

7 Christmore S, Kelly RC, Dashier LA. Improved recovery and stability of

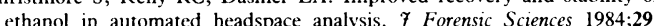
1038-44.

8 Trowbridge EA, Reardon DM, Bradey L, Hutchinson D, Warren CW Automated haematology: construction of univariate reference ranges for blood cell count and size. Med Lab Sci 1989;46:23-32.

9 Gore SM, Altman DG. Statistics in practice. London: British Medical Journal, 1982 .

10 Poupon RE, Papuz L, Sarmini H, Elinck R. A study of the microheterogeneity of transferrin in cirrhotic patients. Clin Chim Acta 1985;151:245-51.

11 Stibler H, Borg S, Joustra M. Micro anion exchange chromatography of carbohydrate deficient transferrin in serum in relation to alcohol 
consumption. Alcoholism: Climical and Experimental Research 1986;10: $535-44$

12 Pequignot G, Chabert C, Eydoux H, Courcowl MA. Augmentation du risque de cirrhose en fonction de la ration d'alcool. Revue de l'Alcoolisme 1974:20:191-202.

13 Wilson P. Drinking hahits in England and Wales. London: HMSO, 1980:11.

14 Van Eijk HG, Van Neort WL, Dubclaar ML, Van Der Heul G. The microheterogeneity of human transferrin in biological fluids. Clin Chim Acto 1983:132:167-71.

15 Chalmers DM, Rinsler MG, MacDermott S, Spicer CC, Levi AJ. Biochemical and hacmatological indicators of excessive alcohol consumption. (iu 981:22:992-6.

16 Chossegros P, Steghens JP, Gravagna B, et al. Serum mitochondrial aspartate aminotransferase (mAST): an additional marker of chronic alcoholism J Hepatol 1988; 7 (suppl):S115.

17 Stibler H, Allgulander C, Borg S, Kiellin KG. Abnormal microheterogencit of transferrin in serum and cerebrospinal fluid in alcoholism. Acta Med Scand 1978;204:49-56.

Accepted 3I Hav 1989)

\title{
Pilot study of treatment of persistent auditory hallucinations by modified auditory input
}

\author{
Mark N Collins, Christine A Cull, \\ Lester Sireling
}

St George's Hospital

Medical School, London

Mark N Collins, MRCPSYCH, lecturer in psychiatry

\section{Springfield Hospital,} London

Christine A Cull, PHD, clinical psychologist

Psychiatric Unit, Barnet General Hospital, Barnet, Hertfordshire EN5 3DJ Lester Sireling, MRCPSYCH, consultant psychiatrist

Correspondence to: Dr Sireling.

BrMed f 1989;299:431-2
Persistent auditory hallucinations occur in several psychiatric conditions. Predictions that hallucinations will decrease with increased external auditory stimulation are supported by several case reports. ${ }^{1.3}$ Green's theory of auditory hallucinations in schizophrenia proposes a defect in interhemispheric transmission of language. ${ }^{+} \mathrm{He}$ suggested that wearing an earplug might reduce verbal input to the non-dominant hemisphere. Studies of this have produced conflicting results.

We assessed the therapeutic potential of various techniques for modifying external auditory input in a patient suffering from intractable auditory hallucinations.

\section{Patient, methods, and results}

The patient was a 53 year old woman who had been an inpatient continuously for 14 months before this study. She had been admitted with delusions and first complained of hearing voices three months after admission. The voices discussed her in the third person and made critical comments about her. They were present for many hours each day. Treatment with neuroleptics, antidepressants, lithium, and carbamazepine failed to suppress them. She had been admitted with psychosis on three previous occasions over 12 years.

She was asked to rate the duration of her auditory hallucinations over a baseline period of seven days. Ratings were made hourly from 9 am to $6 \mathrm{pm}$ with eight point scales and referred to the preceding 30 minutes. Eight experimental conditions were then assessed in random order on alternate days. Each condition was applied for two hours, between midday and $2 \mathrm{pm}$; ratings were made half hourly during the test and hourly afterwards for a further three hours. The test conditions were tapes of pure music (classical), music and speech (BBC Radio 1), boring speech (British Telecom speaking clock), and interesting speech (BBC Radio 4); a blank tape (control); and a hearing aid (right ear); earplug (left ear), and earplug (right ear). A portable cassette tape player with intra-aural headphones was used, allowing continuous play for two hours. All conditions were investigated while she was an inpatient on the same ward, and no other changes were made to her treatment.

She had normal hearing and was right handed. Ratings were consistent over the seven baseline days. The figure shows the results. The tapes of music, music and speech, and interesting speech reduced the hallucinations whereas the blank tape had little effect. The reduction was not sustained beyond the test with music or music and speech, but the effect of interesting speech persisted. An earplug in the left ear made little difference, but when an earplug was used in the right ear the hallucinations disappeared after the test but not during it. Hallucinations temporarily stopped while and after she wore a hearing aid.
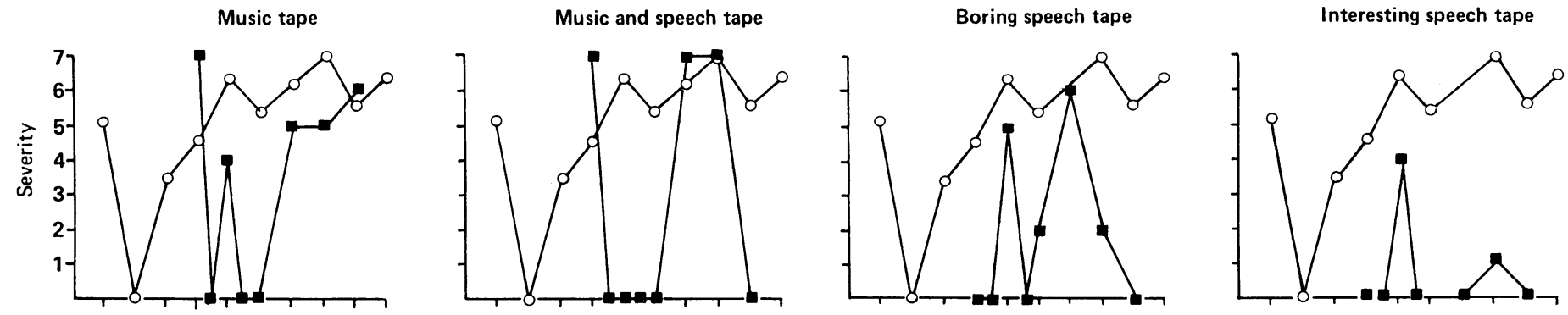

Blank tape

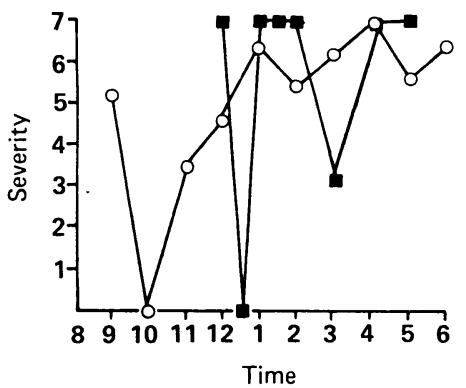

Hearing aid

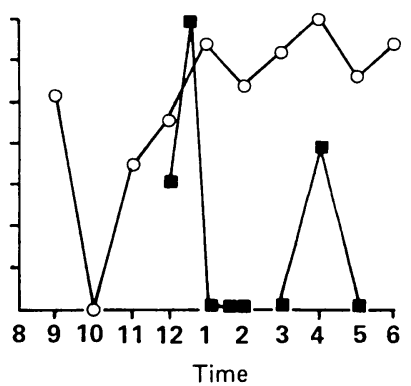

Earplug in left ea

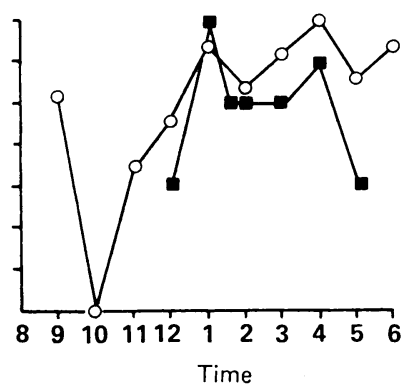

Ratings of hallucinations $(0=$ none, $7=$ continuous $)$ during and after each of eight conditions applied between 12 am and 2 pm. -

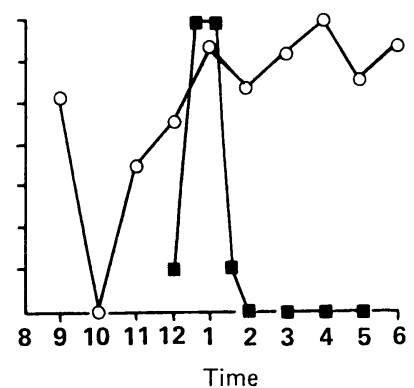

Earplug in right ea 